\title{
Functional conservation of the human EXT1 tumor suppressor gene and its Drosophila homolog tout velu
}

\author{
Ujjaini Dasgupta $\cdot$ Bharat L. Dixit • Melissa Rusch • \\ Scott Selleck • Inge The
}

Received: 30 March 2007 / Accepted: 16 May 2007 /Published online: 4 July 2007

(C) Springer-Verlag 2007

\begin{abstract}
Heparan sulfate proteoglycans play a vital role in signaling of various growth factors in both Drosophila and vertebrates. In Drosophila, mutations in the tout velu (ttv) gene, a homolog of the mammalian EXT1 tumor suppressor gene, leads to abrogation of glycosaminoglycan (GAG) biosynthesis. This impairs distribution and signaling activities of various morphogens such as Hedgehog (Hh), Wingless $(\mathrm{Wg}$ ), and Decapentaplegic (Dpp). Mutations in members of the exostosin (EXT) gene family lead to hereditary multiple exostosis in humans leading to bone outgrowths and tumors. In this study, we provide genetic
\end{abstract}

Communicated by C. Desplan

U. Dasgupta $\cdot$ B. L. Dixit $\cdot$ I. The

Program in Gene Function and Expression,

University of Massachusetts Medical School,

364 Plantation Street,

Worcester, MA 01605, USA

M. Rusch · S. Selleck

Department of Pediatrics, The Developmental Biology Center,

University of Minnesota,

6-160 Jackson Hall, 321 Church Street SE,

Minneapolis, MN 55455, USA

M. Rusch · S. Selleck

Department of Genetics, Cell Biology and Development, The Developmental Biology Center, University of Minnesota, 6-160 Jackson Hall, 321 Church Street SE,

Minneapolis, MN 55455, USA

Present address:

I. The $(\bowtie)$

Division of Developmental Biology, Universiteit Utrecht,

Padualaan 8,

$3584 \mathrm{CH}$ Utrecht, The Netherlands

e-mail: s.i.the@uu.nl and biochemical evidence that the human EXT1 (hEXT1) gene is conserved through species and can functionally complement the ttv mutation in Drosophila. The hEXT1 gene was able to rescue a $t t v$ null mutant to adulthood and restore GAG biosynthesis.

Keywords Drosophila $\cdot$ HSPGs $\cdot$ EXT Conservation · Evolution

\section{Introduction}

Hereditary multiple exostosis (HME) is an autosomal dominant disorder that primarily affects endochondral bone growth (Wicklund et al. 1995) resulting in short stature and formation of benign cartilage-capped tumors (exostosis) in affected individuals (Solomon 1963). Although clinically limb length inequalities, skeletal deformities, and orthopedic complications are common characteristic features, in 2 $5 \%$ of HME patients the benign exostosis transforms to malignant chondrosarcoma or osteosarcoma (Hennekam 1991; Wicklund et al. 1995). Hereditary and sporadic cases of HME have been linked to mutations in the EXT1 and EXT2 genes. The EXT genes encode for type II transmembrane glycoproteins that localize predominantly to the endoplasmic reticulum (ER) and golgi. They form a heterooligomeric complex in the golgi apparatus (Kobayashi et al. 2000; McCormick et al. 2000) and are involved in the synthesis of heparan sulfate glycosaminoglycans (HSGAGs; Lind et al. 1998; McCormick et al. 1998, 2000; Toyoda et al. 2000b; Wei et al. 2000). Heparan sulfate proteoglycans (HSPGs) consist of a glycosylated protein core, which can either be a transmembrane, glycosylphosphatidyl-inositol (GPI)-linked, or secreted protein, and they are abundant on the cell surface and extracellular matrix. 
The Drosophila genome harbors three homologs of the mammalian EXT genes, namely, tout velu (ttv; Bellaiche et al. 1998), sister of tout velu (sotv; Bornemann et al. 2004; Han et al. 2004b), and brother of tout velu (botv; Han et al. 2004b). Mutations in either one of the three genes result in impaired HSPG biosynthesis (The et al. 1999; Han et al. 2004b). Ttv along with Sotv functions in vivo as a HSGAG copolymerase, similar to the vertebrate EXT1 and EXT2 as shown by biochemical and immunohistochemical studies (The et al. 1999; Toyoda et al. 2000a, b; Han et al. 2004b). Interestingly, HSPGs are implicated in shaping the gradient of several morphogens, such as Hedgehog (Hh), Wingless (Wg), and Decapentaplegic (Dpp) by affecting their distribution. In addition, the GPI-linked HSPG Dally has been shown to affect Hh signaling (Desbordes et al. 2003; Han et al. 2004a). Mutations in any of the three Drosophila EXT genes result in impaired $\mathrm{Hh}, \mathrm{Wg}$, and Dpp distribution and signaling (The et al. 1999; Bornemann et al. 2004; Han et al. 2004b; Takei et al. 2004).

Mice lacking Ext1 fail to gastrulate and lack HS biosynthesis (Lin et al. 2000), while mice heterozygous for Ext1 do not show exostoses and express about 50\% HS (Lin et al. 2000). Homozygous Ext2 mice also fail to gastrulate owing to defective HS biosynthesis, and a number of heterozygous animals showed exostoses as well (Stickens et al. 2005). Similar to Ttv, EXT1 has been implicated in Hh distribution and signaling mediated by HSPGs (Lin et al. 2000; Koziel et al. 2004). Interestingly, Indian Hedgehog (Ihh), a mammalian homolog of $\mathrm{Hh}$, plays a pivotal role in controlling the rate of chondrocyte differentiation and bone development (Vortkamp et al. 1996; Zou et al. 1997; Koziel et al. 2004). Lin et al. (2000) have shown that Ihh is incapable of associating with the target cell surface in murine Ext1-/- embryos. Thus it appears that loss of Ext1 leads to impaired Ihh binding and distribution.

To investigate whether human EXT1 $(h E X T 1)$ and Ttv are functionally conserved, we expressed the $h E X T 1$ gene, which is $56 \%$ identical to $t t v$, in Drosophila. We showed that hEXT1 localizes to the ER in Drosophila wing discs and that it interacts biochemically with Sotv in human cell lines to form a complex that functions as the active HSGAG copolymerase. The hEXT1 transgene was able to rescue Drosophila ttv mutants to adulthood and could synthesize HSGAG chains in vivo. Our results highlight the functional conservation between the two orthologues, Drosophila Ttv and human EXT1.

\section{Materials and methods}

\section{EXT construct}

Human EXT1-Green Fluorescent Protein (hEXT1-GFP) was polymerase chain reaction amplified from $\mathrm{pEXT1}$
GFP (McCormick et al. 2000; a gift from Tufaro lab, University of British Columbia), harboring GFP downstream of EXT1 gene, using the primers 5'-GGA CTC AGA TCC CGC AGG ACA CAT-3' and 5'-CCT CTA CAAATG TGG TAT GGC TGA TTATGA-3' and cloned into pGEMT-Easy (Invitrogen) and pUASp2 (a gift from Pernille Rorth) vectors at EcoRI site. The sotv complementary DNA (cDNA) cloned in pAC5.1/V5-His C construct (Han et al. 2004b, a gift from Xinhua Lin's lab). For transfecting human embryonic kidney (HEK) 293 cells $h E X T 1$ cDNA was cloned in p3XFLAG-myc-CMV-26 vector (Sigma) at EcoRI-XbaI site and sotv cDNA in pcDNA4/V5HisB (Invitrogen) at Bam HI-XhoI site.

Fly strains and genetics

Several $h E X T 1-G F P$ transgenic fly strains were generated of which the hEXT1-GFP2.2/Cyo; Dp/TM3 (hEXT1-GFP transgene on the second chromosome) and Sp/Cyo; hEXT1GFP3.1 ( $h E X T 1-G F P$ transgene on the third chromosome) were used. For rescue of homozygous $t t v^{l(2) 00681}$ mutants, the $h E X T 1-G F P 2.2$ transgene was recombined with the $t t v^{l}$ (2)00681 mutation in the FRT $G^{13} t t v^{l(2) 00681} / C y o$ mutant to generate hEXT1-GFP2.2 FRT $G^{13} t^{l(2) 00681} / C y o$; TubulinGal4/TM6B. These flies were then homozygozed to check whether the homozygous $t v^{l(2) 00681}$ mutant could be rescued to adulthood. For ectopic expression of $h E X T 1$ transgene in the engrailed (en) domain, females with enGAL4 on the second chromosome were crossed to males of genotype hEXT1-GFP2.2/Cyo; Dp/TM3.

Females with germline clones (GLCs) lacking maternal and zygotic $t t v^{l(2) 00681}$ activity were generated as described previously (Chou and Perrimon 1996). For expression of $t_{t} v^{l(2) 00681}$ and $h E X T 1-G F P 3.1$ in the hairy-domain females with homozygous $t t v^{l(2) 00681}$ germ-line clones having the genotype $y w f l p^{12} /+$; FRT $G^{13} t^{l(2) 00681} / F R T G^{13} P\left[\right.$ ovo $\left.^{D 1}\right]$; hairy-Gal4/+ were crossed to $t v^{l(2) 00681} / C y o$; UAS-ttv -myc14.1 or $t v^{l(2) 00681} / C y o$; UAS-hEXT1-GFP3.1 males. For sugar chain biochemistry, $h E X T 1-G F P 2.2$ FRT $G^{13} t v^{l(2)}$ ${ }^{00681} / C y o$; tubGal4/SM6 TM6B was generated and nonTubby larvae were used for sugar chain analysis. $t t v^{l(2) 00681}$ mutant used in the study were maintained as a stock over Cyo $P[w+, u b q-G F P]$, the second chromosome balancer marked with GFP and homozygous larvae were identified by the lack of GFP fluorescence detectable under a GFP dissecting microscope.

Antibody staining

Third instar larval imaginal discs were fixed for $20 \mathrm{~min}$ in $4 \%$ formaldehyde in phosphate-buffered saline with $0.1 \%$ Tween20 (PBT). Staining of larval imaginal discs were performed as described before (The et al. 1999). Stainings 
were performed with FM4-64 membrane marker dye (Molecular Probe). Antibodies diluted in PBT include rat anti-Bip, 1:40 (Brabaham Institute, Cambridge, UK), rabbit anti-Lava Lamp, 1:5,000 (Sisson et al. 2000; a gift from John Sisson, University of Texas); mouse anti-HS 3G10, 1:100 (Seikagaku Corporation); mouse anti-V5, 1:5,000 (Invitrogen), and mouse anti-FLAG, 1:5,000 (Sigma). Fixation of embryo and HS GAG staining using 3G10 antibody were performed as described (The et al. 1999). Secondary antibodies for histochemical staining, Western blotting (WB), and fluorescent secondary antibodies were from Jackson Immunoresearch. Images of stained discs were taken with Leica TCS SP2 AOBS Confocal microscope. Images of stained embryos were taken with Zeiss Axioskop 2 Plus microscope. Camera exposure times were kept constant within an experiment.

\section{Immunoprecipitaion and Western blotting}

HEK 293 cells $\left(1 \times 10^{7}\right)$ were transfected with plasmids expressing Flag-tagged hEXT1, V5-tagged Sotv, or both as indicated, using Effectine (Qiagen) following the manufac- turer's procedures. The cells were then lysed in $1.5 \mathrm{ml}$ of lysis buffer $[20 \mathrm{mM}$ Tris- $\mathrm{HCl}(\mathrm{pH} 7.5), 1 \%$ Triton X-100, $150 \mathrm{mM} \mathrm{NaCl}, 5 \mathrm{mM}$ ethylenediamine tetraacetic acid (EDTA), $150 \mu$ Protease Inhibitor Cocktail (Sigma), and $75 \mu$ phenylmethanesulphonylfluoride (PMSF) (Sigma; $10 \mathrm{mg} / \mathrm{ml}$ )] on ice for $20 \mathrm{~min}$. The precleared lysate was used for immunoprecipitation using $5.0 \mu \mathrm{g}$ of either antiHA, anti-Flag, or anti-V5 antibody for $2 \mathrm{~h}$ at $4^{\circ} \mathrm{C}$. Immunoprecipitates were washed five times with wash buffer $[10 \mathrm{mM}$ Tris-HCl (pH 7.5), 0.1\% Triton X-100, $150 \mathrm{mM} \mathrm{NaCl}, 5 \mathrm{mM}$ EDTA, $10 \mu \mathrm{l} / \mathrm{ml}$ of Protease Inhibitor Cocktail (Sigma), and $5 \mu \mathrm{l} / \mathrm{ml}$ PMSF $(10 \mathrm{mg} / \mathrm{ml})$ ] and analyzed by WB. WB was carried out as described (The et al. 1999). Antibodies used were mouse anti-V5, 1:5,000 (Invitrogen) and mouse anti-FLAG, 1:5,000 (Sigma).

Sugar chain biochemistry

Analyses of HS-derived disaccharides were performed as previously reported (Toyoda et al. 2000a) using 100 third instar larvae. As a wild type strain, Oregon $\mathrm{R}$ was used. Results shown are from two independent experiments.
Fig. 1 hEXT1-GFP localizes to ER and golgi in Drosophila melanogaster. Wing imaginal disc cells, showing the expression of hEXT1-GFP indicated by presence of GFP (green) (a, d, and $\mathbf{g}$ ), stained with membrane dye FM4-64 (red) (b), the golgi marker Lavalamp (red) (e), and ER marker Bip (red) (h). hEXT1-GFP does not colocalize with FM4-64 (c).

Colocalization of hEXT1-GFP with Lavalamp (f) or Bip (i) is seen as yellow. Arrows indicate examples where hEXT1-GFP colocalization with golgi marker protein Lavalamp is detected (f)
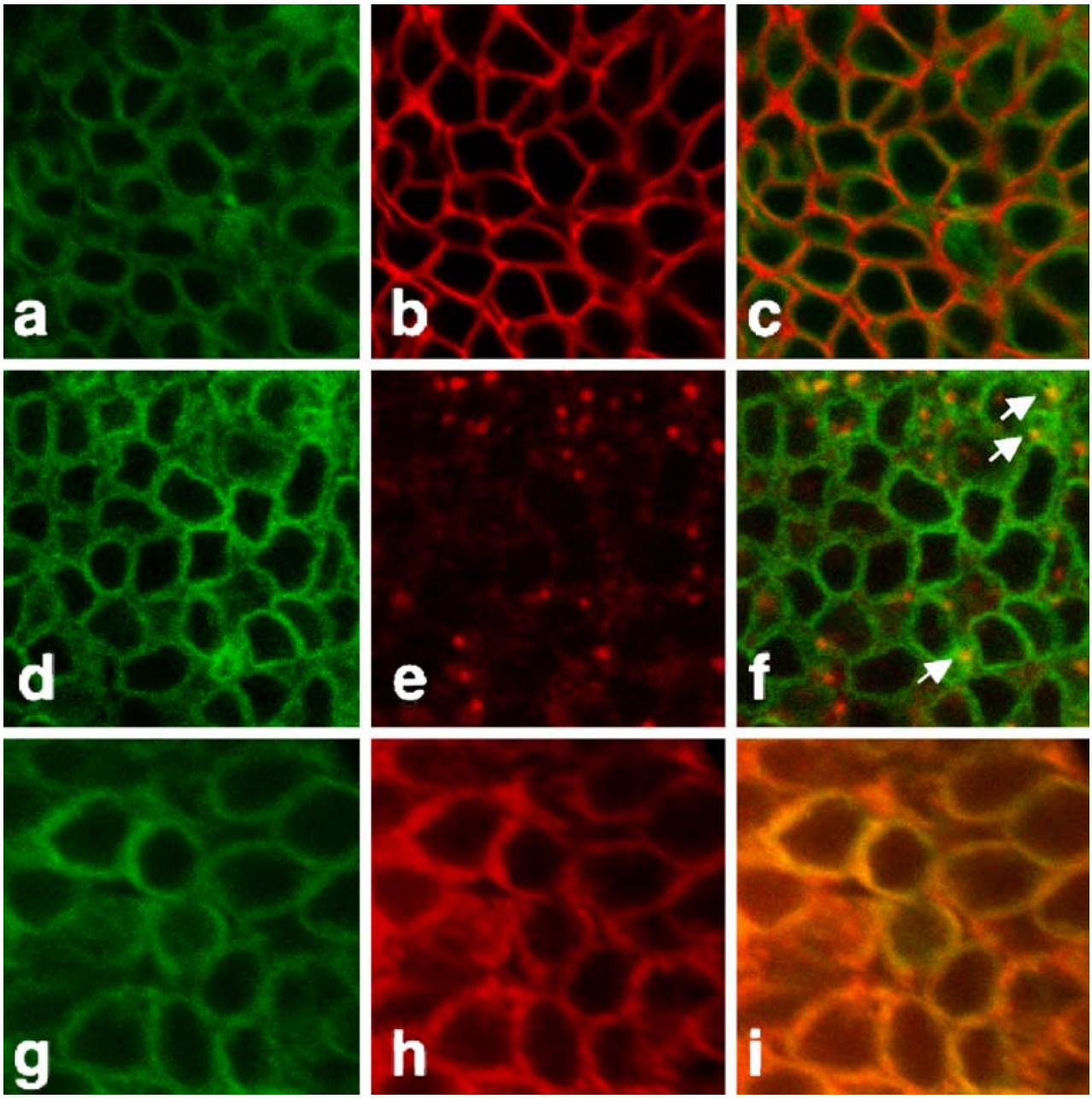
Table 1 Rescue of a ttv mutant with $h E X T 1-G F P$ transgene

\begin{tabular}{lllll}
\hline & $\begin{array}{l}\text { Experiment } \\
\text { number }\end{array}$ & $\begin{array}{l}\text { Total } \\
\text { number of } \\
\text { flies }\end{array}$ & $\begin{array}{l}\text { Total number } \\
\text { of rescued } \\
\text { flies }\end{array}$ & $\begin{array}{l}\text { Percent of } \\
\text { rescued } \\
\text { flies }\end{array}$ \\
\hline Control & 1 & 42 & 0 & 0 \\
& 2 & 25 & 0 & 0 \\
& 3 & 49 & 0 & 0 \\
Rescue & 1 & 58 & 4 & 6.9 \\
& 2 & 37 & 2 & 5.4 \\
& 3 & 63 & 3 & 4.7 \\
\hline
\end{tabular}

As control animals, $t t v$ mutant flies that do not harbor the $h E X T 1-G F P$ were used, while in the rescue experiment, ttv mutant flies expressing the $h E X T 1-G F P$ were used. The percentage of rescued flies indicate the percentage of total animals rescued to adulthood.

\section{Results}

\section{Subcellular localization of hEXT1 protein in Drosophila}

In both Drosophila and mammals, EXT1 or EXT2 protein localizes mainly in the ER (McCormick et al. 1998, 2000; The et al. 1999; Han et al. 2004b; Kobayashi et al. 2000). However, when both the proteins were expressed in the same cell, the EXT proteins were found to be mostly relocated from the ER to the golgi network (McCormick et al. 2000) where polymerization and sulfation of HSPG occurs (Muckenthaler et al. 1998). To determine the subcellular localization of hEXT1-GFP in Drosophila, the wing imaginal discs expressing hEXT1-GFP under the control of en-GAL4 were stained for colocalization with plasma membrane marker dye FM4-64 (Fig. 1a-c), ER protein Bip (Fig. 1d-f), and golgi protein Lava Lamp (Fig. 1g-i). hEXT1-GFP colocalized mostly with Bip (Fig. 1f) and partially with Lava Lamp (Fig. 1i) but not with membrane dye FM4-64 (Fig. 1c). These results show that hEXT1-GFP protein in Drosophila localizes mainly to the ER and partially to the golgi, as previously shown in case of EXT1 in human cells and Ttv in Drosophila (McCormick et al. 1998, 2000; The et al. 1999; Han et al. 2004b), indicating that the protein localizes as endogenous Ttv.

Rescue of homozygous $t t v$ mutants by expression of $h E X T 1$

Homozygous $t t v^{l(2) 00681}$ null mutants (from here on $t t v$ ) die at the pupal stage, and animals depleted from maternal product are embryonic lethal. However, when the hEXT1-

Fig. 2 HSPG biosynthesis in $t t v$ mutant embryos is rescued by $h E X T 1-G F P$ expression in vivo. Staining of wild-type (WT) (a, b), $t$ tv mutant (c), $t t v$ mutant expressing $t t v-m y c$ transgene in the hairy-domain (d), and $t t v$ mutant expressing $h E X T 1-G F P$ transgene in the hairy-domain (e, f) with 3G10 antibody. No staining is detected in the negative controls where WT embryos (a) or ttv mutants expressing $h E X T 1-G F P(\mathbf{e})$ were not treated with heparinase III. Anterior is to the left in all the panels
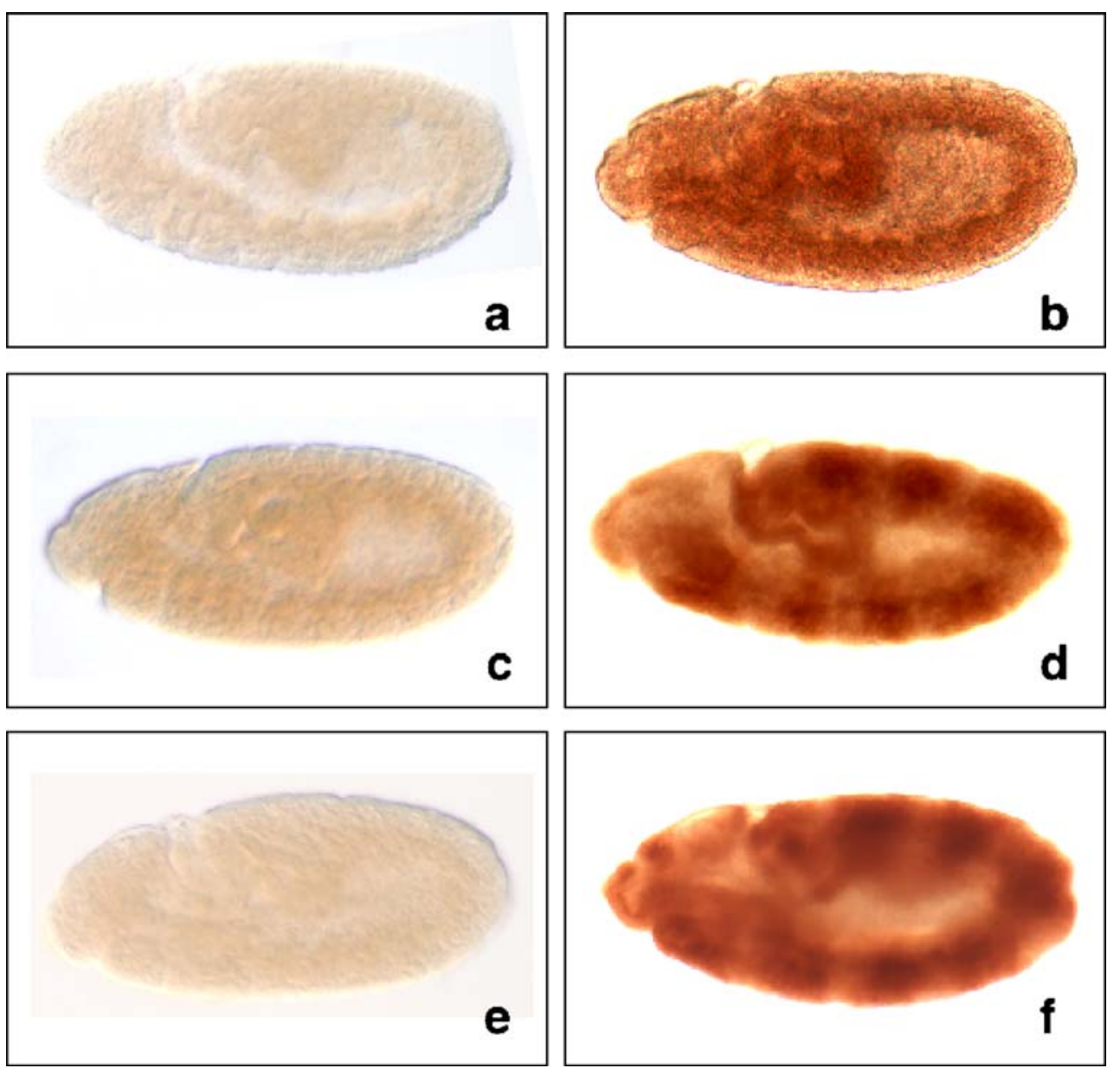
GFP transgene was expressed in homozygous ttv mutants using tub-GAL4 as the driver, rescue of ttv mutants to adulthood was achieved. In three independent experiments ( $n=158), 5.7 \%$ of total animals developed to adult flies (Table 1). Interestingly, the rescued flies did not show any abnormal change in phenotype and are comparable to wild type control flies.

HS biosynthesis is restored in ttv germline clones by $h E X T 1$

In Drosophila embryos, HSPG biosynthesis is abrogated in the absence of Ttv activity (The et al. 1999), and in vertebrates, the EXT proteins function similarly in the HSPG biosynthesis pathway (Lind et al. 1998; McCormick et al. 1998; Toyoda et al. 2000a, b). We asked whether the $h E X T 1$ transgene is able to effectively restore HSPG biosynthesis in homozygous $t t v$ mutant embryos. To detect HSPGs in vivo, we stained embryos with an anti-HS antibody (3G10) that detects unsaturated glucoronate at the nonreducing ends of HS chains after digestion of HSPGs with heparinase III (David et al. 1992; The et al. 1999). Staining with 3 G10 showed a uniform pattern in WT embryos (Fig. 2b), whereas no staining was detected in embryos that were not treated with heparinase III (Fig. 2a). When 3G10 staining was tested in the ttv mutant embryos, a strong reduction in staining intensity was observed (Fig. 2c) that was regained when wild type ttv activity was restored in the hairy-domain (Fig. 2d) as was previously shown (The et al. 1999). Interestingly, strong and specific staining was seen as well in embryos where the ttv activity was restored by $h E X T 1-G F P$ transgene expressed in the hairy-domain (Fig. 2f) indicating that the $h E X T 1-G F P$ transgene is functional in homozygous $t t v$ embryos. Thus, the hEXT1-GFP transgene, as a true ortholog, can substitute the activity of the ttv gene and restore HS biosynthesis in Drosophila.

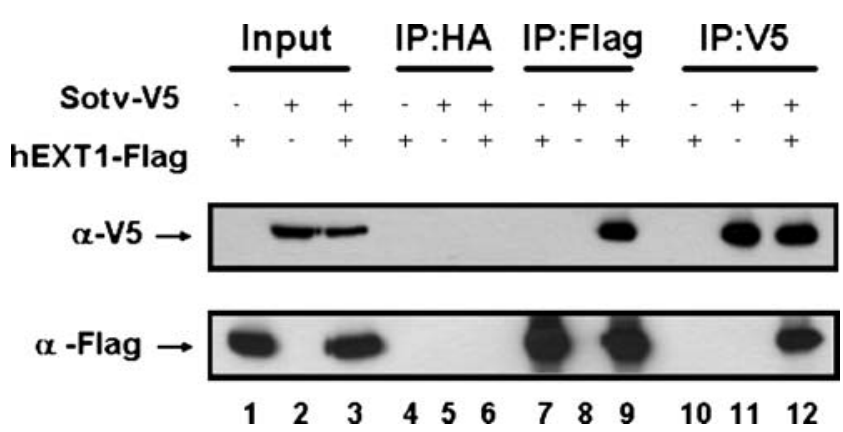

Fig. 3 Co-immunoprecipitation of hEXT1-GFP and Sotv. HEK 293 cells were transfected with plasmids expressing Flag-tagged hEXT1, V5-tagged Sotv, or both as indicated. The cell lysates were immunoprecipitated with either anti-HA as a negative control (lane 4-6), anti-Flag (lane 7-9), or anti-V5 (lane 10-12) antibody and then analyzed by WB with mouse anti-V5 ( $\alpha$-V5, upper panel) or mouse anti-Flag $(\alpha$-Flag, lower panel $)$
Ttv protein interacts with hEXT1

Biochemical studies have shown that the vertebrate EXT1 and EXT2 can associate to form biologically functional hetero-oligomeric complex that exhibits glycosyltransferase activity in which both subunits are essential for full activity (Kobayashi et al. 2000; McCormick et al. 2000; Senay et al. 2000; Wei et al. 2000; Zak et al. 2002). Han et al. (2004b) have shown that Ttv and Sotv behave similarly in Drosophila. As hEXT1-GFP is able to restore HS biosynthesis in homozygous ttv mutants, we wanted to see whether it can interact biochemically with Sotv to reconstitute a functional HSGAG copolymerase. Therefore, we cotransfected FLAG tagged hEXT1-GFP and V5-tagged Sotv into HEK293 cells. Upon immunoprecipitation with anti-V5 or anti-FLAG antibody, we could detect Sotv interacting with hEXT1-GFP (Fig. 3). V5-tagged Sotv is seen to co-immunoprecipitate with hEXT1-Flag (lane 9, upper panel) and Flag-tagged hEXT1 with Sotv-V5 (lane 12, lower panel). This biochemical interaction shows that hEXT1-GFP is able to interact with Sotv in Drosophila and together they function as an active HSGAG polymerase. Similar interaction could also be reproduced in Drosophila Schneider's S2 cells transfected with hEXT1GFP and V5-Sotv (data not shown). These data suggest that hEXT1 is capable of performing a similar function as Ttv, suggesting that HS biosynthesis in insects and vertebrates is conserved.

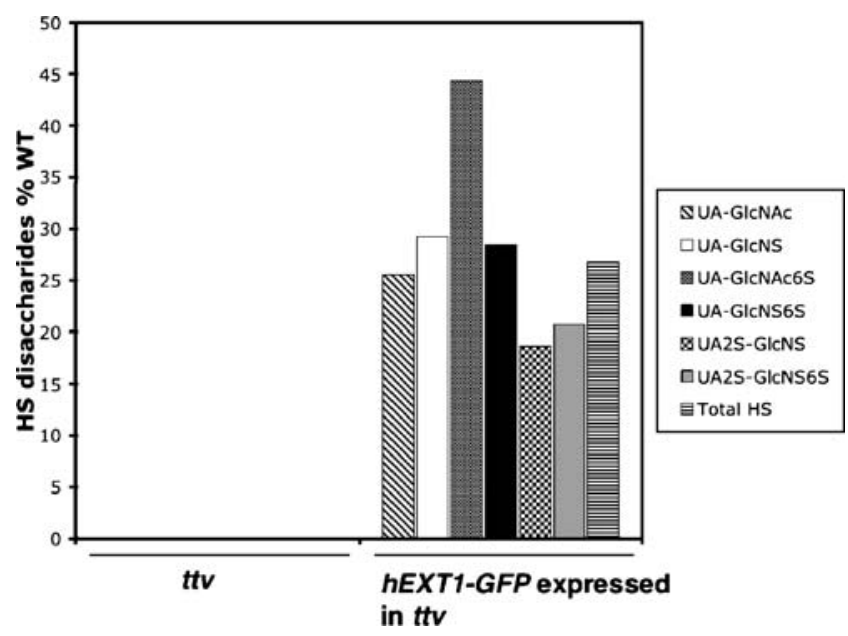

Fig. 4 Biosynthesis of HS-derived disaccharides is restored in $t t v$ mutants expressing $h E X T 1-G F P$. HS-derived disaccharides from ttv and ttv mutants expressing $h E X T 1-G F P$ transgene, normalized to the dry weight of the sample, are shown as a percentage of WT controls. The six different bars represent disaccharides profiled $\triangle \mathrm{UA}-\mathrm{GlcNAc}$, $\Delta$ UA-GlcNS, $\Delta$ UA-GlcNA6S, $\Delta$ UA-GlcNS6S, $\Delta$ UA-2S-GlcNS, and $\Delta \mathrm{UA} 2 \mathrm{~S}-\mathrm{GlcNs} 6 \mathrm{~S}$. The total HS content in the rescued $t$ tv mutants is $26.6 \%$ 
hEXT1-GFP interacts with Sotv and synthesizes HS in vivo

The $t t v$ null mutants have undetectable levels of HS-derived disaccharides (Toyoda et al. 2000a, b). We next addressed whether hEXT1-GFP and Sotv can function as biologically active HS copolymerases to synthesize HS in homozygous ttv null mutants. Therefore, we evaluated the levels of HSderived disaccharides in ttv mutant larvae expressing $h E X T 1-G F P$ transgene. We found that the ttv mutant animals expressing the $h E X T 1-G F P$ transgene could synthesize significant amounts of all the HS-derived disaccharides $(25.5 \% \Delta$ UA-GlcNAc, $29.2 \% \Delta$ UA-GlcNS, $44.3 \% \quad \Delta$ UA-GlcNA6S， 28.4\% $\Delta$ UA-GlcNS6S， $18.6 \%$ $\Delta$ UA-2S-GlcNS, and $20.7 \% \Delta$ UA2S-GlcNs6S of wild type) in comparison to the ttv null mutants (Fig. 4), which showed no detectable traces of any of these HS-derived disaccharides. Thus, the $\mathrm{hEXT1}$ gene can effectively substitute the function of ttv in Drosophila, synthesizing $26.6 \%$ of total HS-derived disaccharides in vivo.

\section{Discussion}

Previous studies have shown that the EXT gene family is required for HSGAG biosynthesis in Drosophila and vertebrates. We were able to rescue the ttv null mutation in Drosophila using the human orthologue EXT1. The lower than expected percentage of rescued animals might be due to either a difference between the expression levels of the $h E X T 1-G F P$ transgene and wild type $t t v$ or temporal differences in expression. The $t t v$ gene is ubiquitously expressed in Drosophila embryos and larval tissues, therefore the tubulin promoter used to express the transgene is expected to mimic the expression pattern. However, we do not expect that the partial rescue will change the interpretation of the experiments as we could detect rescue of the HSPG synthesis in ttv mutant animals expressing the $h E X T 1-G F P$ transgene.

The hEXT1-GFP protein localizes in the ER in Drosophila wing imaginal disc cells as previously reported in human cell lines. In addition, we have been able to show interaction of hEXT1-GFP with Sotv, the EXT2 homologue, as previously shown for EXT1 in human cells and Ttv in Drosophila. The hEXT1-GFP and Sotv heterodimer most likely forms an active enzymatic complex as GAG synthesis of HSPGs is restored. Thus, we have demonstrated a functional conservation of HS copolymerase between insects and vertebrates.

HSPGs have been shown to affect distribution and signaling of several secreted growth factors, including FGF, Wnt, TGF $\beta$, and Hh family members in Drosophila and vertebrates (Perrimon and Bernfield 2000; Lin and Perrimon 2002; Nybakken and Perrimon 2002). Studies carried out with Ext1 and Ext2 null mice show that HSPGs are required for developing embryos to survive and that haploinsufficiency of HS is the main cause of exostoses (Koziel et al. 2004; Stickens et al. 2005). Interestingly, Ihh and other growth factors affect bone development and Ihh protein is absent from the surface of Ext1 deficient mice cells (Lin et al. 2000; Koziel et al. 2004).

We might therefore hypothesize that the absence of the Ttv orthologue EXT1 leads to an abrogation of HSGAG biosynthesis, and this would translate into altered signaling of Ihh and/or other growth factors. The growth factors are involved in chondrocyte differentiation and proliferation and alteration of their signaling could lead to formation of exostosis (Hopyan et al. 2002; Koziel et al. 2004). As EXT1 and EXT2 are copolymerases, HME arising from loss of EXT2 may result from the same molecular mechanism. The functional conservation of hEXT1 in Drosophila opens up possibilities of using Drosophila as an in vivo system to distinguish silent polymorphisms from inactivating mutations of the altered EXT genes from human HME patients.

Acknowledgments We thank Peter S. Bak and Hau Hung for generating the hEXT1-GFP lines. We thank Frank Tufaro, Pernille Rorth, Xinhua Lin, and John Sisson for sending us reagents. We also thank the UMass Medical School Drosophila community for interesting discussions and suggestions. This work was funded by grants from the National Institutes of Health (GM066220 to IT and GM54832 to SBS).

\section{References}

Bellaiche Y, The I, Perrimon N (1998) Tout-velu is a Drosophila homologue of the putative tumour suppressor EXT-1 and is needed for Hh diffusion. Nature 394:85-88

Bornemann DJ, Duncan JE, Staatz W, Selleck S, Warrior R (2004) Abrogation of heparan sulfate synthesis in Drosophila disrupts the Wingless, Hedgehog and Decapentaplegic signaling pathways. Development 131:1927-1938

Chou TB, Perrimon N (1996) The autosomal FLP-DFS technique for generating germline mosaics in Drosophila melanogaster. Genetics 144:1673-1679

David G, Bai XM, Van der Schueren B, Cassiman JJ, Van den Berghe H (1992) Developmental changes in heparan sulfate expression: in situ detection with mAbs. J Cell Biol 119:961-975

Desbordes SC, Sanson B (2003) The glypican dally-like is required for Hedgehog signaling in the embryonic epidermis of Drosophila. Development 130:6245-6255

Han C, Belenkaya TY, Wang B, Lin X (2004a) Drosophila glypicans control the cell-to-cell movement of Hedgehog by a dynaminindependent process. Development 131:601-611

Han C, Belenkaya TY, Khodoun M, Tauchi M, Lin X (2004b) Distinct and collaborative roles of Drosophila EXT family proteins in morphogen signalling and gradient formation. Development 131:1563-1575

Hennekam RC (1991) Hereditary multiple exostoses. J Med Genet 28:262-266 
Hopyan S, Gokgoz N, Poon R, Gensure RC, Yu C, Cole WG, Bell RS, Juppner H, Andrulis IL, Wunder JS, Alman BA (2002) A mutant PTH/PTHrP type I receptor in enchondromatosis. Nat Genet 30:306-310

Kobayashi S, Morimoto K, Shimizu T, Takahashi M, Kurosawa H, Shirasawa T (2000) Association of EXT1 and EXT2, hereditary multiple exostoses gene products, in Golgi apparatus. Biochem Biophys Res Commun 268:860-867

Koziel L, Kunath M, Kelly OG, Vortkamp A (2004) Ext1-dependent heparan sulfate regulates the range of Ihh signaling during endochondral ossification. Dev Cell 6:801-813

Lin X, Perrimon N (2002) Developmental roles of heparan sulfate proteoglycans in Drosophila. Glycoconj J 19:363-368

Lin X, Wei G, Shi Z, Dryer L, Esko JD, Wells DE, Matzuk MM (2000) Disruption of gastrulation and heparan sulfate biosynthesis in EXT1-deficient mice. Dev Biol 224:299-311

Lind T, Tufaro F, McCormick C, Lindahl U, Lidholt K (1998) The putative tumor suppressors EXT1 and EXT2 are glycosyltransferases required for the biosynthesis of heparan sulfate. J Biol Chem 273:26265-26268

McCormick C, Leduc Y, Martindale D, Mattison K, Esford LE, Dyer AP, Tufaro F (1998) The putative tumour suppressor EXT1 alters the expression of cell-surface heparan sulfate. Nat Genet 19:158-161

McCormick C, Duncan G, Goutsos KT, Tufaro F (2000) The putative tumor suppressors EXT1 and EXT2 form a stable complex that accumulates in the Golgi apparatus and catalyzes the synthesis of heparan sulfate. Proc Natl Acad Sci USA 97:668-673

Muckenthaler M, Gray NK, Hentze MW (1998) IRP-1 binding to ferritin mRNA prevents the recruitment of the small ribosomal subunit by the cap-binding complex eIF4F. Mol Cell 2:383-388

Nybakken K, Perrimon N (2002) Heparan sulfate proteoglycan modulation of developmental signaling in Drosophila. Biochim Biophys Acta 1573:280-291

Perrimon N, Bernfield M (2000) Specificities of heparan sulphate proteoglycans in developmental processes. Nature 404:725-728

Senay C, Lind T, Muguruma K, Tone Y, Kitagawa H, Sugahara K, Lidholt K, Lindahl U, Kusche-Gullberg M (2000) The EXT1/ EXT2 tumor suppressors: catalytic activities and role in heparan sulfate biosynthesis. EMBO Rep 1:282-286
Sisson JC, Field C, Ventura R, Royou A, Sullivan W (2000) Lava Lamp, a novel peripheral golgi protein, is required for Drosophila melanogaster cellularization. J Cell Biol 151:905-917

Solomon L (1963) Hereditary multiple exostosis. J Bone Jt Surg Am 45:292-304

Stickens D, Zak BM, Rougier N, Esko JD, Werb Z (2005) Mice deficient in Ext2 lack heparan sulfate and develop exostoses. Development 132:5055-5068

Takei Y, Ozawa Y, Sato M, Watanabe A, Tabata T (2004) Three Drosophila EXT genes shape morphogen gradients through synthesis of heparan sulfate proteoglycans. Development 131:73-82

The I, Bellaiche Y, Perrimon N (1999) Hedgehog movement is regulated through tout velu-dependent synthesis of a heparan sulfate proteoglycan. Mol Cell 4:633-639

Toyoda H, Kinoshita-Toyoda A, Fox B, Selleck SB (2000a) Structural analysis of glycosaminoglycans in animals bearing mutations in sugarless, sulfateless, and tout-velu. Drosophila homologues of vertebrate genes encoding glycosaminoglycan biosynthetic enzymes. J Biol Chem 275:21856-21861

Toyoda H, Kinoshita-Toyoda A, Selleck SB (2000b) Structural analysis of glycosaminoglycans in Drosophila and Caenorhabditis elegans and demonstration that tout-velu, a Drosophila gene related to EXT tumor suppressors, affects heparan sulfate in vivo. J Biol Chem 275:2269-2275

Vortkamp A, Lee K, Lanske B, Segre GV, Kronenberg HM, Tabin CJ (1996) Regulation of rate of cartilage differentiation by Indian hedgehog and PTH-related protein. Science 273:613-622

Wei G, Bai X, Gabb MM, Bame KJ, Koshy TI, Spear PG, Esko JD (2000) Location of the glucuronosyltransferase domain in the heparan sulfate copolymerase EXT1 by analysis of Chinese hamster ovary cell mutants. J Biol Chem 275:27733-27740

Wicklund CL, Pauli RM, Johnston D, Hecht JT (1995) Natural history study of hereditary multiple exostoses. Am J Med Genet 55:43-46

Zak BM, Crawford BE, Esko JD (2002) Hereditary multiple exostoses and heparan sulfate polymerization. Biochim Biophys Acta 1573:346-355

Zou H, Wieser R, Massague J, Niswander L (1997) Distinct roles of type I bone morphogenetic protein receptors in the formation and differentiation of cartilage. Genes Dev 11:2191-2203 